\title{
Impact of COVID-2019 on stroke services in China: survey from the Chinese Stroke Association
}

Xia Wang (D) , ${ }^{1}$ Menglu Ouyang, ${ }^{1}$ Cheryl Carcel, ${ }^{1}$ Chen Chen, ${ }^{2}$ Lingli Sun ${ }^{(1)},{ }^{3}$
Jie Yang (D) , ${ }^{4}$ Yao Zhang, ${ }^{5}$ Guofang Chen, ${ }^{6}$ Shoujiang You, ${ }^{7}$ Yongjun Cao, ${ }^{7}$ Lu Ma, ${ }^{8}$
Xin Hu, ${ }^{8}$ Yi Sui (D) ${ }^{5}$ Craig Anderson, ${ }^{1,3,9}$ Lili Song, ${ }^{3}$ Yongjun Wang, ${ }^{10,11}$
David Wang (D) ${ }^{12}$

To cite: Wang $X$, Ouyang $M$, Carcel C, et al. Impact of COVID-2019 on stroke services in China: survey from the Chinese Stroke Association. Stroke \& Vascular Neurology 2020;5: e000514. doi:10.1136/ svn-2020-000514

- Additional material is published online only. To view, please visit the journal online (http://dx.doi.org/10.1136/svn2020-000514).

$\mathrm{XW}$ and $\mathrm{MO}$ contributed equally.

Received 17 July 2020 Revised 28 August 2020 Accepted 2 September 2020 Published Online First 28 September 2020

Check for updates

(c) Author(s) (or their employer(s)) 2020. Re-use permitted under CC BY-NC. No commercial re-use. See rights and permissions. Published by BMJ.

For numbered affiliations see end of article.

Correspondence to

Dr Lili Song;

Isong@georgeinstitute.org.cn

Professor Yongjun Wang, Department of Neurology, Beijing Tiantan Hospital, Beijing, China;

yongjunwang111@aliyun.com

\section{ABSTRACT}

Background The COVID-19 pandemic and physical distancing guidelines have compelled stroke practices worldwide to reshape their delivery of care significantly. We aimed to illustrate how the stroke services were interrupted during the pandemic in China.

Methods A 61-item questionnaire designed on Wenjuanxing Form was completed by doctors or nurses who were involved in treating patients with stroke from 1 February to 31 March 2020.

Results A total of 415 respondents completed the online survey after informed consent was obtained. Of the respondents, $37.8 \%, 35.2 \%$ and $27.0 \%$ were from mild, moderate and severe epidemic areas, respectively. Overall, the proportion of severe impact (reduction $>50 \%$ ) on the admission of transient ischaemic stroke, acute ischaemic stroke (AIS) and intracerebral haemorrhage (ICH) was $45.0 \%, 32.0 \%$ and $27.5 \%$, respectively. Those numbers were $36.9 \%, 27.9 \%$ and $22.3 \% ; 36.5 \%, 22.1 \%$ and $22.6 \%$; and $66.4 \%, 47.5 \%$ and $41.1 \%$ in mild, moderate and severe epidemic areas, respectively (all $p<0.0001$ ). For AIS, thrombolysis was moderate ( $20 \%-50 \%$ reduction) or severely impacted (>50\%), as reported by $54.4 \%$ of the respondents, while thrombectomy was $39.3 \%$. These were $44.4 \%, 26.3 \%$; $44.2 \%, 39.4 \%$; and $78.2 \%, 56.5 \%$, in mild, moderate and severe epidemic areas, respectively (all $p<0.0001$ ). For patients with acute ICH, 39.8\% reported the impact was severe or moderate for those eligible for surgery who had surgery. Those numbers were $27.4 \%$, $39.0 \%$ and $58.1 \%$ in mild, moderate and severe epidemic areas, respectively. For staff resources, about $20 \%$ (overall) to $55 \%$ (severe epidemic) of the respondents reported moderate or severe impact on the on-duty doctors and nurses.

Conclusion We found a significant reduction of admission for all types of patients with stroke during the pandemic. Patients were less likely to receive appropriate care, for example, thrombolysis/thrombectomy, after being admitted to the hospital. Stroke service in severe COVID-19 epidemic areas, for example, Wuhan, was much more severely impacted compared with other regions in China.

\section{INTRODUCTION}

The COVID-19 pandemic represents an unprecedented global healthcare challenge. It has reached 188 countries and regions with nearly 24 million confirmed cases and 820000 deaths globally. ${ }^{1}$ It is becoming clear that the impact of COVID-19 on health and healthcare system goes beyond the disease it produces, as health systems have to adapt and cope with the need to provide care to other non-communicable conditions. ${ }^{2}$

China has been successful in fighting COVID-19 since late January as the COVID-19 epidemic first broke out in Wuhan and spread rapidly to the rest of China. ${ }^{1}$ Patients with stroke are a particularly high-risk COVID-19 clinical group by vascular risk factors and critical illness. During the COVID-19 pandemic, many hospitals have been implementing prevention and management protocols to provide quality and uninterrupted care for patients with stroke. ${ }^{3}$ However, it remains unclear how the stroke services were interrupted from the provider's perspective during the COVID-19 pandemic. We examined the impact of COVID-19 on stroke services in China using data from a cross-sectional survey. We also aimed to find out if epidemic levels (mild, moderate and severe; Wuhan vs others) were proportional to the differences in stroke care interruption.

\section{METHODS}

\section{Study design}

In this cross-sectional study, a 61-item survey was administered to doctors or nurses who were involved in treating patients with stroke from 1 February to 31 March 2020. They must be familiar with the information of this study's interest, namely, overall hospital organisation and infection control in response to COVID19, stroke pathway, patient admission and management, and staff resources from 1 February to 31 March 2020.

\section{Sampling}

We advertised via the China Stroke Association website and official WeChat account and 
Chinese Stroke Centre Alliance platform. To achieve the representativeness in terms of epidemic level, we proactively approached healthcare providers in some provinces to encourage the participation: severe epidemic areas: Hubei (confirmed cases of 68129 to 10 May 2020) and Guangdong (1589); moderate epidemic areas: Jiangsu (653) and Sichuan (561); and mild epidemic areas: Shanxi (198) and Liaoning (146). We also proactively approached neurosurgeons to encourage participation as patients with acute spontaneous intracerebral haemorrhage (ICH) are managed under the Department of Neurosurgery in some hospitals to ensure the study covers all the stroke subtypes.

A clinical research associate at the George Institute for Global Health has made contact with Intensive Care Bundle with Blood Pressure Reduction in Acute Cerebral haemorrhage Trial (INTERACT3, NCT03209258) recruitment sites. Site principal investigators (PIs) who were senior directors of departments were invited to complete the questionnaire as they have a better command of the overall situation within the department.

\section{Dataset description and questionnaire design}

The questionnaire consisted of 61 questions with 41 made mandatory. There were four sections: general information, overall hospital organisation and infection control in response to COVID-19, patients with stroke admission and management during COVID-19 and staff resourcing. The first general information section contained five questions that covered hospital location, care level of a hospital and the information on the department. The second section on infection control was to understand how personal protective equipment (PPE) was implemented to protect staff. ${ }^{4}$ The third section on patient admission and management contained questions on major facets of the stroke care pathway ${ }^{5}$ such as early appropriate cerebral imaging $(<1$ hour) and rapid thrombolysis and referral for thrombectomy. The last section on staff resourcing was to

A Hospital preparedness in response to COVID-19

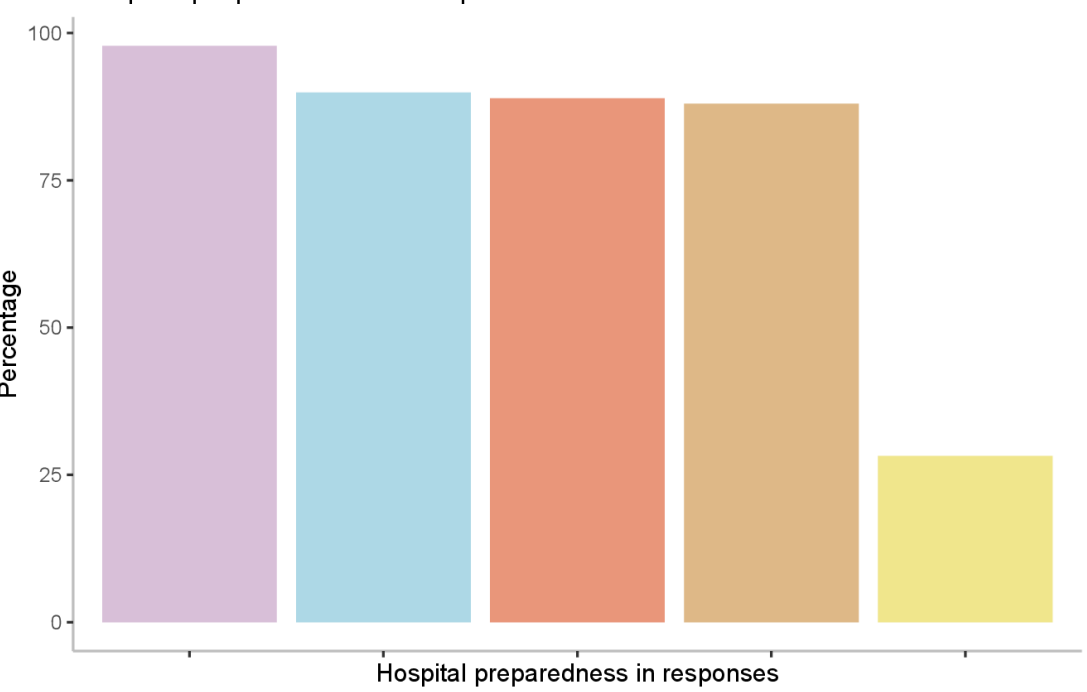

Hospital preparedness in responses

Admission after rule out the possibility of COVID19 Chest CT performed before admission to stroke unit Designated areas to manage suspected COVID-19 patients Protocols was implemented to deal with COVID-19 RT-PCR test performed before admission to stroke unit

B Hospital PPE supply in response to COVID-19

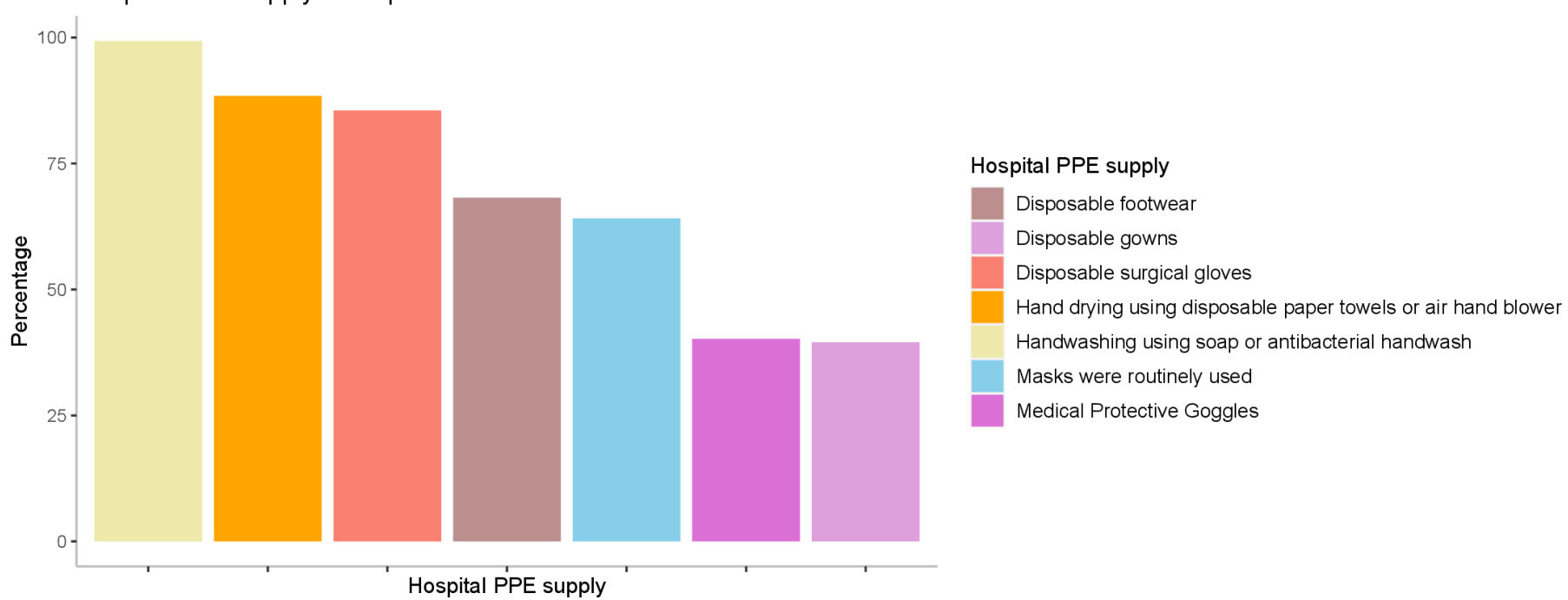

Figure 1 Overall hospital organisation in response to COVID-19. PPE, personal protective equipment. 
A. Average time to be treated by a stroke specialist and to CT scan

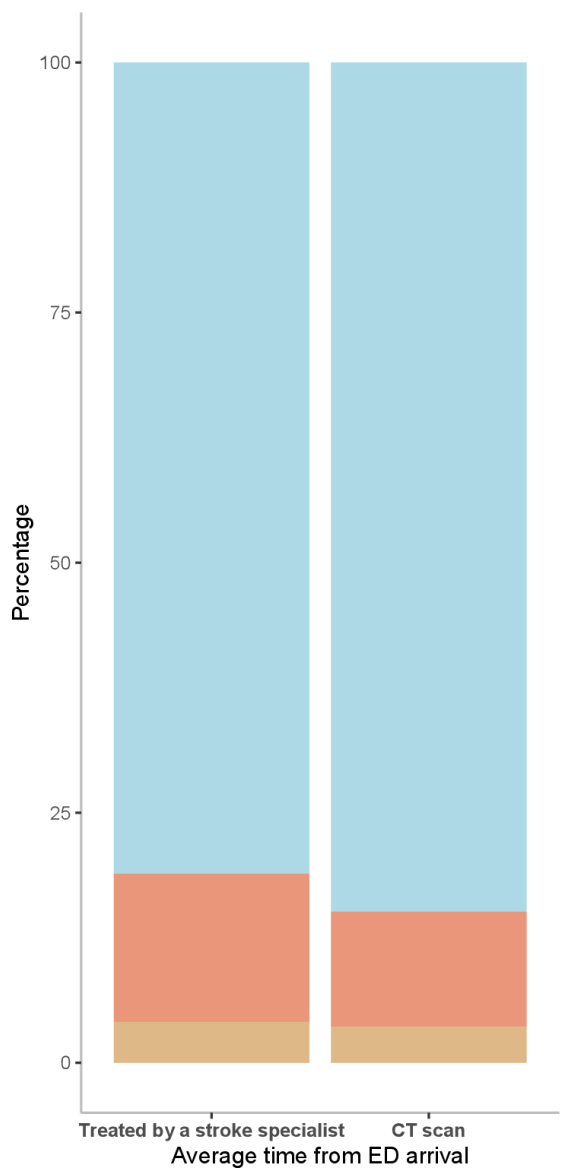

B. DNT and DPT delay for AIS patients

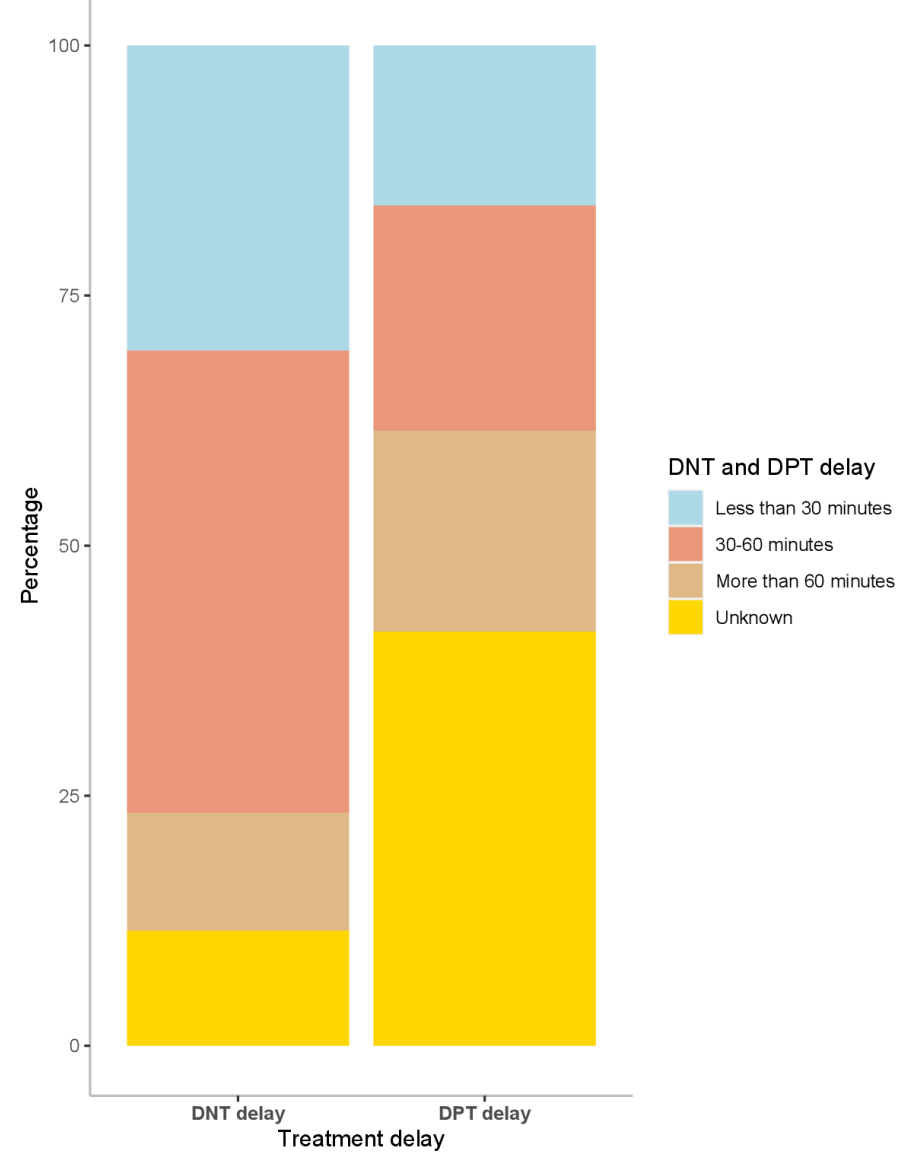

Figure 2 Impact of COVID-19 on treatment delay. AIS, acute ischaemic stroke; DNT, door-to-needle time; DPT, door to puncture; ED emergency department.

understand how the stroke unit staff was redeployed due to COVID-19 and if their actual working hours were reduced. All information was collected on a virtual platform, the Wenjuanxing online system.

Authors XW and MO independently conducted comprehensive literature reviews to identify pertinent questions that assessed the impact of COVID-19 and agreed on a final list of items included in the survey questions, which were then pretested and finalised. This step was to ensure the validity and reliability of the questionnaire. We also invited seven neurologists and neurosurgeons to test the questionnaire to ensure the feasibility. No personal information (eg, names, birthday and email) was collected.

\section{Data analysis}

Data were downloaded from Wenjuanxing Forms into Microsoft Excel and subsequently coded and transferred into the SAS V.9.4. Descriptive statistics were calculated in the form of frequencies, and comparison was made using $\chi^{2}$ or Fisher exact test where appropriate.

\section{RESULTS}

General characteristics of the respondents

A total of 415 respondents completed the online survey after informed consent was obtained. Of the respondents,
$27.0 \%, 35.2 \%$ and $37.8 \%$ were from severe, moderate and mild epidemic areas, respectively. Over $80 \%$ were from tertiary teaching hospitals in the urban areas, while one-half were from COVID-19-designated hospitals. About $74.5 \%$ and $18.6 \%$ were from the departments of neurology and neurosurgery, respectively (online supplemental table 1). We collected 85 questionnaires from INTERACT3 recruitment sites.

\section{Overall hospital organisation and infection control in response to COVID-19 pandemic}

Nearly all of the hospitals have implemented their own protocols to deal with the crisis from February to March 2020. About half of the stroke units had single bedrooms and three-quarters had two to four bedrooms. About $88 \%$ had designated areas to manage patients with fever, and $99.3 \%$ had handwashing using soap or liquid sanitiser in their hospitals. Only $64 \%$ reported mask supply was enough, and the proportion of accessing sufficient disposable surgical gloves, footwear, gowns and goggles was $85.5 \%, 68.2 \%, 39.5 \%$ and $40.2 \%$, respectively. About $88.9 \%$ and $28.2 \%$ reported that chest CT and nucleic acid testing for COVID-19 were performed before admission to the stroke unit, respectively (online supplemental table 1 and figure 1 ). 


\section{Admission of patients with stroke}

About one-third of the respondents reported that the number of suspected patients with stroke who presented to the emergency department (ED) by ambulance was severely impacted (defined as reduction more than $50 \%$ ) from February to March 2020, compared with the same period last year. Although 50\% of the respondents reported treatment delay in ED, $80 \%$ of them still reported that early appropriate cerebral imaging and assessment by stroke specialists could be accessed within 1 hour on ED arrival (figure 2).

Online supplemental figure 1 shows the extent of reduced admission of patients with stroke by stroke subtypes from February to March 2020, compared with the same period last year. The proportion of very severe (almost no patients) or severe impact (reduction $>50 \%$ ) on transient ischaemic attack (TIA), acute ischaemic stroke (AIS) and ICH was $45.0 \%, 32.0 \%$, and $27.5 \%$, respectively.

The proportion of very severe or severe impact on stroke (all subtypes) admission was significantly higher in COVID-19-designated hospitals compared with nondesignated hospitals (online supplemental table 2), and in severe epidemic areas compared with mild or moderate areas (figure 3 and online supplemental table 3).

\section{Management of patients with stroke}

For AIS, $54.4 \%$ of the respondents reported thrombolysis for AIS was moderate or severely impacted (defined as reduction $20 \%-50 \%$ and $>50 \%$, respectively). The most common reasons were missing the therapeutic window on hospital arrival or too many tests for ruling out COVID19. Of the respondents, $11.8 \%$ reported the delay in doorto-needle time of more than 1 hour. Of the respondents, $39.3 \%$ reported thrombectomy was moderate or severely impacted with similar reasons for thrombolysis and $20.1 \%$ reported the delay in door-to-puncture time of more than 1 hour. For acute ICH, 39.8\% reported the impact was severe or moderate for those eligible for surgery who had surgery; $44.9 \%$ reported this was due to deployment of staff, so surgery could not be performed; $24.9 \%$ reported surgery room was not open. More than $60 \%$ of the

\section{A. Severe epidemic}
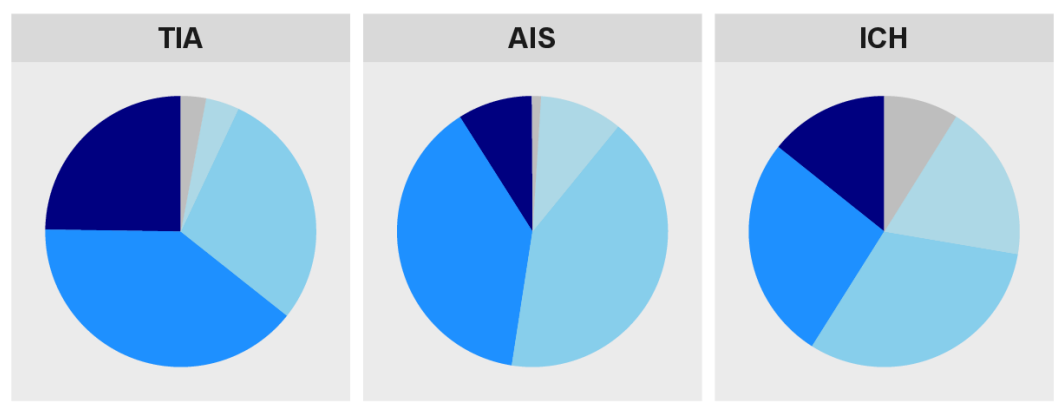

\section{B. Moderate epidemic}
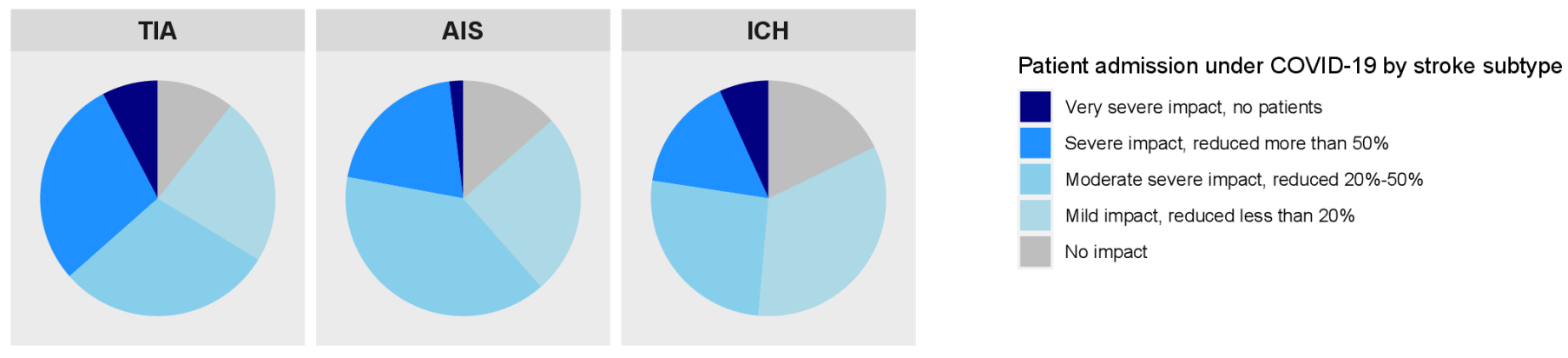

\section{Mild epidemic}

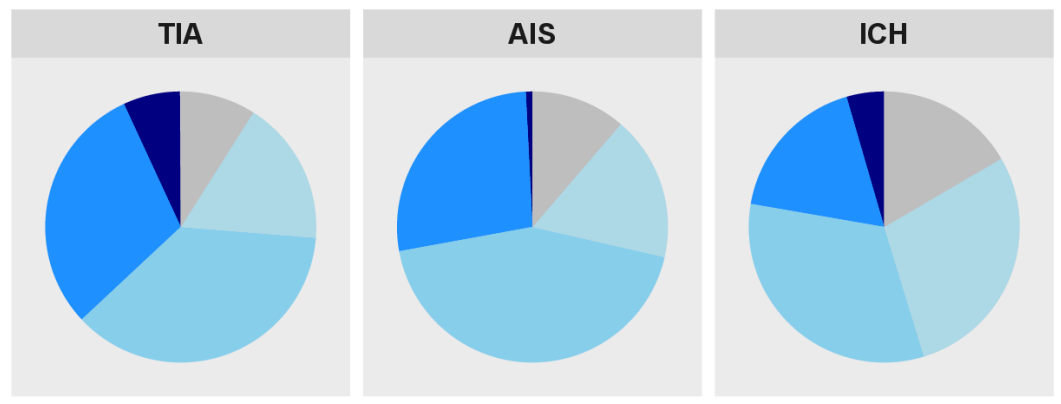

Figure 3 Impact of COVID-19 on patient admission by stroke subtype and epidemic levels. AIS, acute ischaemic stroke; ICH, intracerebral haemorrhage; TIA, transient ischaemic attack. 


\section{A. Severe epidemic}

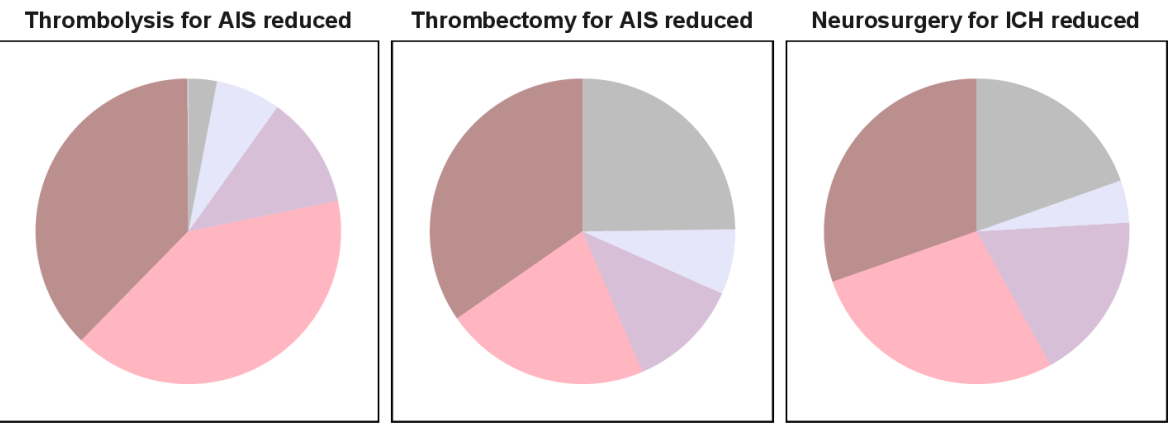

\section{B. Moderate epidemic}

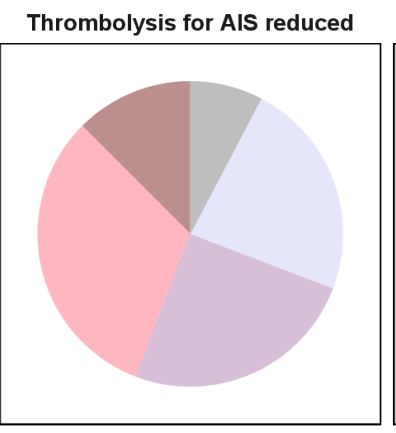

Thrombectomy for AIS reduced
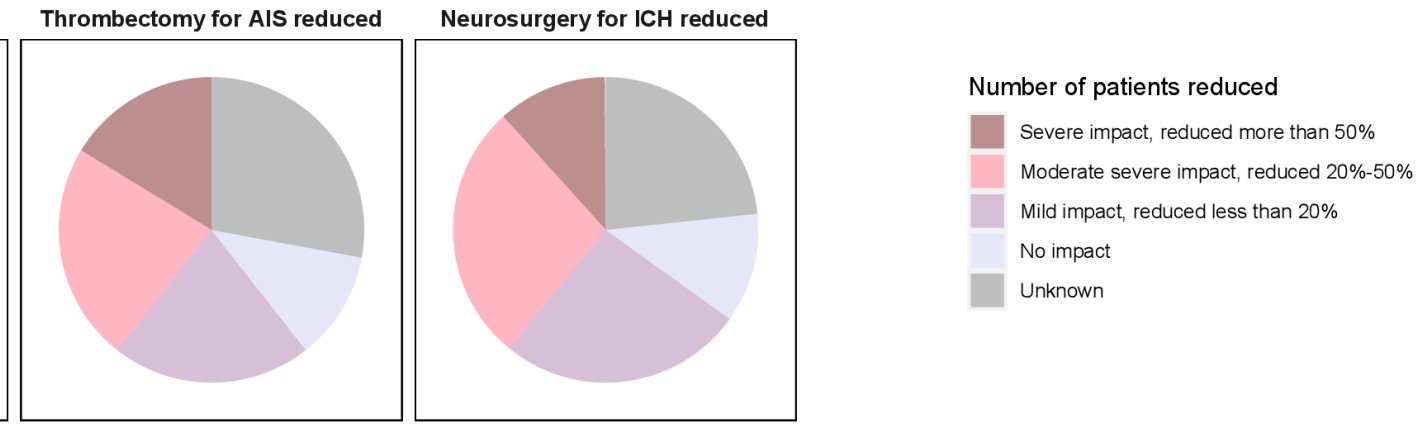

\section{Mild epidemic}

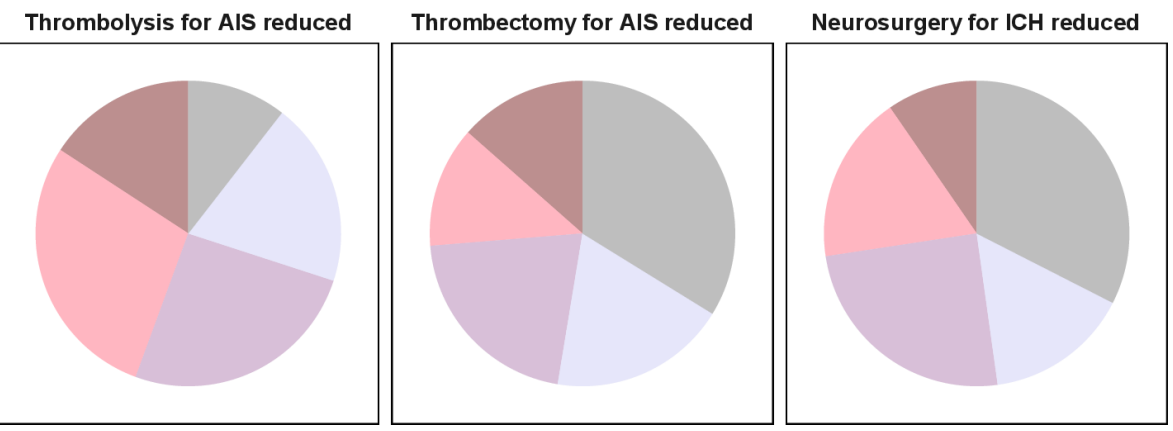

Figure 4 Extent of acute treatments reduced during COVID-19 pandemic by epidemic levels. AIS, acute ischaemic stroke; $\mathrm{ICH}$, intracerebral haemorrhage; TIA, transient ischaemic attack.

responders reported no increase in complications. About $1 / 3$ reported the hospital stay was lengthened, and only a half reported that patients with stroke could be followed up as scheduled (online supplemental figure 2).

Those acute management were more severely impacted in COVID-19-designated hospitals compared with nondesignated hospitals (online supplemental table 2), and in the severely impacted provinces compared with mild or moderate areas (figure 4 and online supplemental table $3)$.

\section{Staff resources}

About $20 \%$ of the responders reported that on-duty doctors and nurses were moderately or severely impacted (reduction of more than 20\%) from February to March 2020, compared with the same period last year. The most common reason was medical staff being reallocated to support the fever clinic and COVID-19 ward, or going to Hubei Province. Over $60 \%$ reported that the multidisciplinary team was intact, including early rehabilitation, nutrition and mental health support (online supplemental figure 3).

Similarly, the staff resourcing was also more likely to be impacted in COVID-19-designated hospitals compared with non-designated hospitals (online supplemental table 2 ), and in the severely impacted provinces compared with mild or moderate areas (figures 5 and 6 and online supplemental table 3).

\section{Sensitivity analysis in Wuhan}

From February to March 2020, among 24 respondents from Wuhan, very severe (almost no patients) or severe impact (reduction $>50 \%$ ) on TIA, AIS and ICH of $94.7 \%$, $84.2 \%$ and $83.3 \%$, respectively, was reported. About $80 \%$ reported treatment delay in the ED. The proportion of early appropriate cerebral imaging $(<1$ hour $)$ and assessment by stroke specialists ( $<1$ hour) was performed 


\section{A. Severe epidemic}

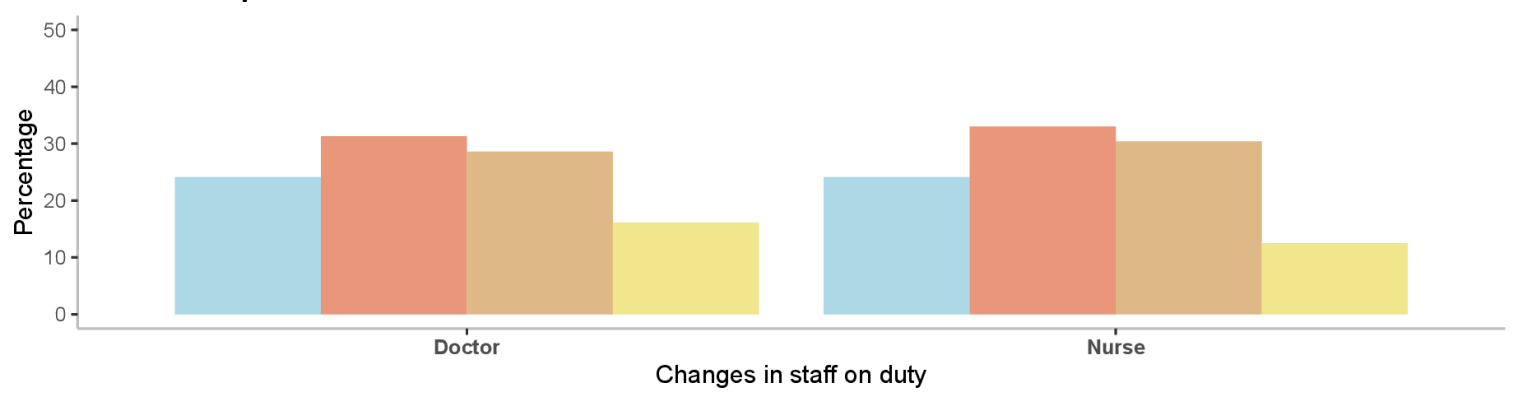

B. Moderate epidemic

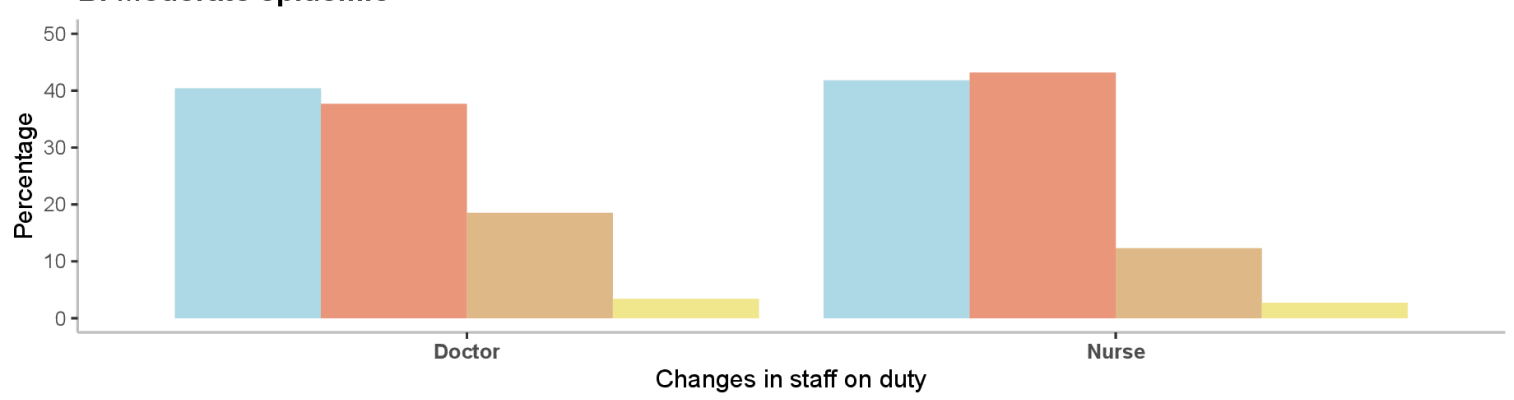

Changes in staff on duty

No change

Decrease $<20 \%$

Decrease 20\%-50\%

Decrease $>50 \%$

\section{Mild epidemic}

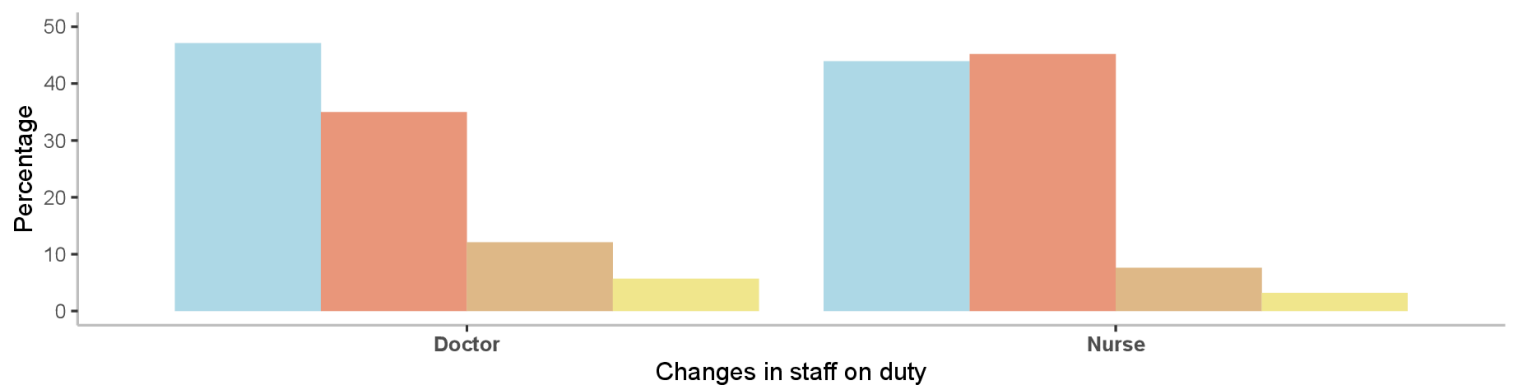

Figure 5 Impact of COVID-19 on staff resourcing by epidemic levels.

in $52.6 \%$ and $36.8 \%$ of patients with AIS, respectively (online supplemental table 2).

For AIS, $89.5 \%$ reported that thrombolysis was severely $(>50 \%)$ or moderately $(20 \%-50 \%)$ impacted; the most common reason was missing the therapeutic window on hospital arrival. About $73.7 \%$ reported that thrombectomy was severely or moderately impacted. The most common reasons were the deployment of staff, so surgery could not be performed, and the surgery room was not open. For acute ICH, 75\% reported the impact was severe or moderate for those eligible for surgery who had surgery (online supplemental figure 4).

About $80 \%$ of the responders reported the number of on-duty doctors and nurses moderately or severely reduced from February to March 2020. Only 25\% reported that a multidisciplinary team was still retained, including early rehabilitation, nutrition and mental health.

\section{Subgroup analysis to investigate the robustness of the data} A subgroup analysis was performed by if conducted in the INTERACT3 recruitment sites (online supplemental table 1 ). This analysis did not show any statistical differences for the major facets: the number of admitted patients with AIS $(p=0.454)$, the number of admitted patients with acute ICH ( $\mathrm{p}=0.129)$; thrombolysis rate reduction $(\mathrm{p}=0.752)$; and thrombectomy rate reduction $(\mathrm{p}=0.485)$.

\section{DISCUSSION}

In this cross-sectional survey, we found (1) excellent hospital preparedness to deal with the COVID-19 pandemic crisis; (2) overall, the proportion of severe impact (reduction $>50 \%$ ) on the admission of TIA, AIS and ICH was $45.0 \%, 32.0 \%$ and $27.5 \%$, respectively, and more patient admission reduction was seen in the more severely impacted areas; (3) for AIS, thrombolysis was moderately $(20 \%-50 \%$ reduction) or severely impacted $(>50 \%)$, as reported by $54.4 \%$ of the respondents, while thrombectomy was $39.3 \%$; for patients with acute ICH, $39.8 \%$ reported the impact was severe or moderate for those eligible for surgery who had surgery; similarly, less effective acute treatment was provided in the more severely impacted areas; and (4) for staff resources, about $20 \%$ (overall) of the respondents reported moderate or severe impact on the on-duty doctors and nurses, and more reductions were seen the more severely impacted areas. 


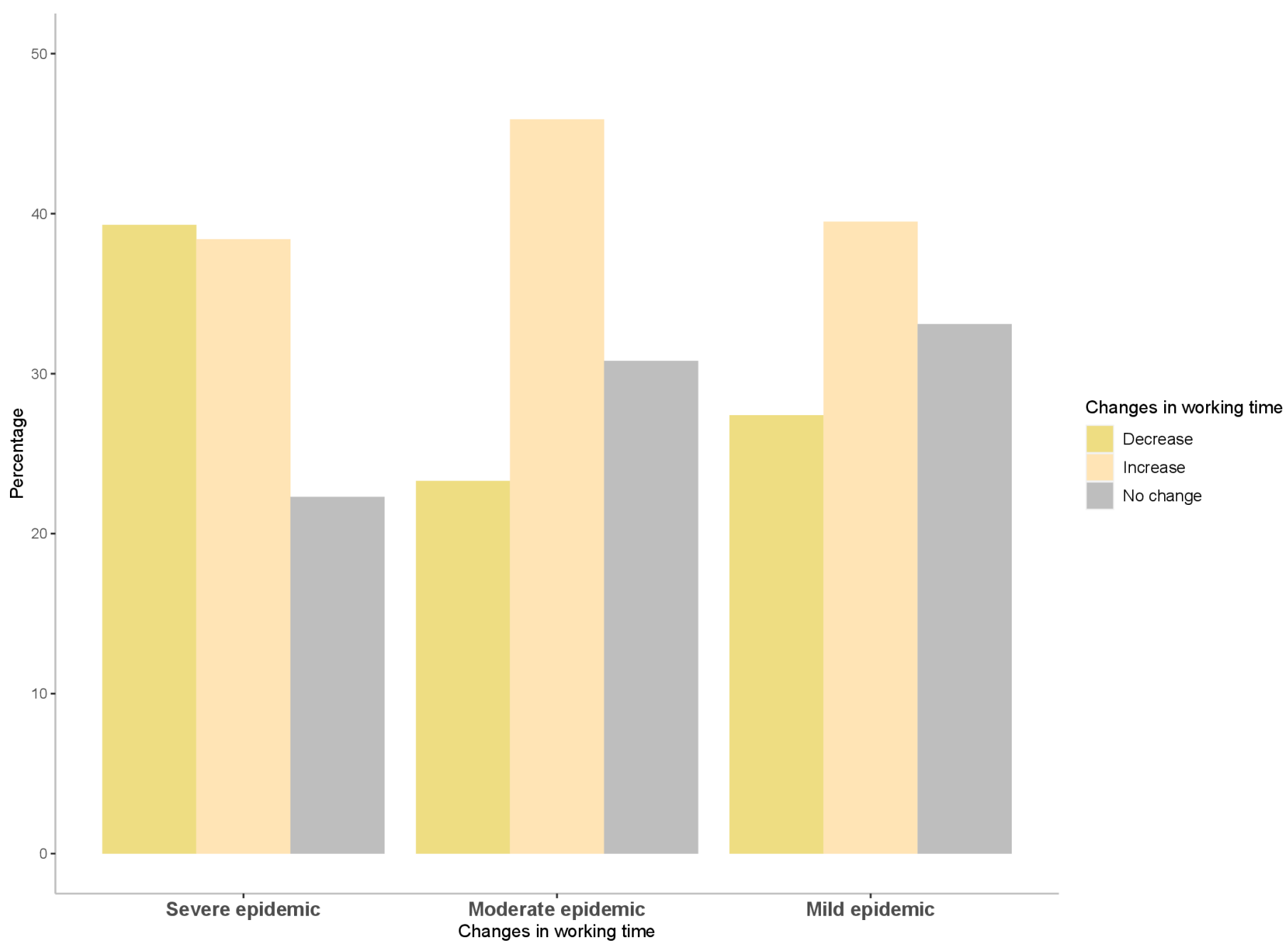

Figure 6 Impact of COVID-19 on working hours by epidemic levels.

Our findings are consistent with those from a big data analysis of 280 hospitals across China. ${ }^{6}$ In the prehospital setting, the admission of TIA has experienced the biggest drop, followed by AIS, and ICH had the smallest decline. For in-hospital care, the COVID-19 outbreak has impacted the capacity to provide stroke care, resulting in a significant reduction in services such as thrombolysis, thrombectomy and neurosurgical procedures for ICH. Notably, thrombolysis has experienced a higher drop compared with thrombectomy and neurosurgery. Further to findings from the big data analysis, ${ }^{6}$ we have provided information on how staff resources were reallocated and how PPE was implemented to protect staff during the pandemic. Our data have quantified the differences between different epidemic levels and COVID-19-designated hospitals versus non- designated hospitals, which present a reallife picture of how COVID-19 interrupted stroke services. Furthermore, we have provided data on acute ICH and neurosurgery, which have not been reported previously.

Our data suggest that stroke service was much more severely impacted in severe COVID-19-infected areas, for example, Wuhan compared with others. This is consistent with global findings that the impact of COVID-19 on neurological services and patients is profound. ${ }^{7}$ The COVID-19 pandemic and physical distancing guidelines have compelled stroke practices worldwide to reshape their delivery of care significantly. Simultaneously, nonurgent surgical procedures and medical appointments have been cancelled to increase bed capacity and to care for patients with the infection, while many neurologists have had to leave their clinics to help other physicians on the frontlines. In Wuhan, the same as Italy, ${ }^{8}$ hospitals have been divided into COVID-19+ and COVID-free ones; many stroke units and neurointensive care units were reorganised or even closed, and many neurologists have been reallocated to the care of patient with COVID19. These measures have had a significant impact on the organisation of the usual emergency networks for stroke. The reorganisation and centralised stroke care also resulted in a reduced number of stroke units and hospitals, which contributed to the reduced thrombolysis or thrombectomy for AIS and neurosurgery care for acute ICH. We also found that almost half of the respondents reported a severe impact of COVID-19 on seeking medical attention by patients with TIA, AIS and ICH. This might be explained by the fact that patients with TIA or 
strokes remained at home due to the fear of getting an infection if going to the hospital during the outbreak. ${ }^{68}$

We found that overall hospitals did well in protecting healthcare personnel and infection control. With the comprehensive policy in place, hospitals tried to deliver uninterrupted care to all in need. ${ }^{3}$ Almost all the hospitals have released their own official policies on COVID-19 prevention and control; $88 \%$ reported a designated location, separated from other clinical areas for the treatment and triage of possible COVID-19. About 90\% reported that a chest CT would be performed to rule out the possibility of COVID-19 before admission to a stroke unit.

In our study, the targeted selection of the provinces by the epidemic level was used to examine how the stroke services performed or were interrupted according to the severity of the epidemic of COVID-19. However, there are some limitations to this study. First, this was an anonymous online survey that raised the concern of data accuracy. However, we compared data from INTERACT3 and nonINTERACT3 recruitment sites, and no major differences have been identified. We approached INTERACT3 site PIs who are all senior directors and have a good command of the situation within the department to complete the questionnaires, so the data are more likely to be reliable. Second, there might be duplicated responses from the same hospital, which would be hard to differentiate. However, the 85 questionnaires from INTERACT3 sites were collected from individual hospitals, and again no difference was identified between INTERACT3 and nonINTERACT3 sites. Besides, we did not collect any information regarding the respondent due to the requirement to be anonymous.

In conclusion, there was a clear drop in admission to the hospital of all types of patients with stroke. Patients with stroke were also less likely to receive timely care if admitted during the pandemic. Stroke service in the severe epidemic areas was much more impacted by COVID-19 compared with moderate/low areas in China.

\section{Author affiliations}

${ }^{1}$ The George Institute for Global Health, Faculty of Medicine, University of New South Wales, Sydney, New South Wales, Australia

${ }^{2}$ Department of Neurology, Shanghai East Hospital, Shanghai, Shanghai, China ${ }^{3}$ Stroke Division, The George Institute China at Peking University Health Science Centre, Beijing, PR China, Shanghai, China

${ }^{4}$ Department of Neurology, The First Affiliated Hospital of Chengdu Medical College, Chengdu, China

${ }^{5}$ Department of Neurology, Shenyang Brain Hospital, Shenyang Medical College, Shenyang, China

${ }^{6}$ Department of Neurology, Xuzhou Central Hospital, Xuzhou, Jiangsu, China ${ }^{7}$ Department of Neurology, The Second Affiliated Hospital of Soochow University, Suzhou, Jiangsu, China

${ }^{8}$ Department of Neurosurgery, West China Hospital of Medicine, Chengdu, Sichuan, China

${ }^{9}$ Department of Neurology, Royal Prince Alfred Hospital, Sydney, NSW, Australia

${ }^{10}$ Department of Neurology, China National Clinical Research Center for Neurological Disease, Beijing, China
${ }^{11}$ Department of Neurology, Tiantan Clinical Trial and Research Center for Stroke, Beijing, China

${ }^{12}$ Neurovascular Division, Department of Neurology, Barrow Neurological Institute, St. Joseph's Hospital and Medical Center, Phoenix, Arizona, USA

Contributors XW, M0, LS,YW, DW and CA contributed to study concept and overall design; CCa, LS, JY, YZ, GC, SY, YC, LM, HX, YS and CCh contributed to the questionnaire development and data acquisition. XW and $\mathrm{MO}$ contributed to data analysis and manuscript writing. All authors critically reviewed and approved the manuscript.

Funding The authors have not declared a specific grant for this research from any funding agency in the public, commercial or not-for-profit sectors.

Competing interests CA reports receiving fees for Advisory Panels of Astra Zeneca and Medtronic, speaking at seminars for Takeda China and Boehringer Ingelheim, and a research grant from Takeda China.

Patient consent for publication Not required.

Ethics approval Considering the low risk and anonymity of the participants, we simply obtained the informed consent. After reading the opening statement at the beginning of the questionnaire, the respondents would be directed to answer questions only if they are willing to participate. Otherwise, the survey would be ended. Data collection complied with Wenjuanxing terms and conditions.

Provenance and peer review Commissioned; externally peer reviewed. Data availability statement Data are available upon reasonable request. Individual deidentified participant data used in these analyses can be shared by formal request with protocol and statistical analysis plan from any qualified investigator to the Research Office of The George Institute for Global Health, Australia. A tailored dataset specific to the research question will be shared for 6 months, and the data can be only accessed by qualified statisticians for the proposed analysis.

Open access This is an open access article distributed in accordance with the Creative Commons Attribution Non Commercial (CC BY-NC 4.0) license, which permits others to distribute, remix, adapt, build upon this work non-commercially, and license their derivative works on different terms, provided the original work is properly cited, appropriate credit is given, any changes made indicated, and the use is non-commercial. See: http://creativecommons.org/licenses/by-nc/4.0/.

\section{ORCID iDs}

Xia Wang http://orcid.org/0000-0002-1684-7076

Lingli Sun http://orcid.org/0000-0003-3859-9672

Jie Yang http://orcid.org/0000-0002-9032-5348

Yi Sui http://orcid.org/0000-0002-2898-7877

David Wang http://orcid.org/0000-0003-2277-4608

\section{REFERENCES}

1 John Hopkins Unversity. Coronavirus resource center. Available: https://coronavirus.jhu.edu/[Accessed 25/08/2020].

2 Emanuel EJ, Persad G, Upshur R, et al. Fair allocation of scarce medical resources in the time of Covid-19. N Engl J Med 2020;382:2049-55.

3 Wang X, Chen Y, Li Z, et al. Providing uninterrupted care during COVID-19 pandemic: experience from Beijing Tiantan Hospital. Stroke Vasc Neurol 2020;5:180-4.

4 CDC. Comprehensive Hospital preparedness checklist for coronavirus disease, 2019. Available: https://www.cdc.gov/coronavirus/2019ncov/hcp/hcp-hospital-checklist.html

$5 \mathrm{NHS}$ clinical guide for the management of stroke patients during the coronavirus pandemic. Available: https://www.england.nhs.uk/ coronavirus/wp-content/uploads/sites/52/2020/04/C0033-_Specialtyguide_-Stroke-and-coronavirus-V1-update_16-April-003.pdf

6 Zhao J, Li H, Kung D, et al. Impact of the COVID-19 epidemic on stroke care and potential solutions. Stroke 2020;51:1996-2001.

7 The Lancet Neurology. The neurological impact of COVID-19. Lancet Neurol 2020;19:471.

8 Bersano A, Pantoni L. On being a neurologist in Italy at the time of the COVID-19 outbreak. Neurology 2020;94:905-6. 